\title{
Variable Power Broadcasting in Ad Hoc Networks
}

\author{
Avinash Chiganmi, Kamil Sarac, and Ravi Prakash \\ Department of Computer Science, University of Texas at Dallas \\ Email: \{avinash.chiganmi@student.,ksarac@, ravip@\}utdallas.edu
}

\begin{abstract}
Network wide broadcast is a frequently used operation in ad hoc networks and consumes significant amount of energy. Reducing the overall power consumption is extremely important in increasing the longevity of ad hoc networks. As a result, developing energy efficient broadcast operations becomes an important issue in ad hoc networks. In this paper, we propose an adaptive power broadcast algorithm that uses localized information in ad hoc networks. Our algorithm uses a novel technique to improve energy efficiency in network wide broadcast. Our simulation based comparisons show the superiority of our algorithm compared to other approaches.
\end{abstract}

\section{INTRODUCTION}

Network wide broadcast is a frequently used operation in ad hoc networks. In addition to data broadcasting, it is used for various protocol operations including route discover and address assignment. Nodes in ad hoc networks work with limited battery power and the efficient utilization of this power is important for increasing the lifetime of the individual nodes as well as the overall network. Depending on the size and topology of an ad hoc network, a network wide broadcast may require multiple nodes to participate in the dissemination of the messages to the entire network. Hence, network wide broadcast is an energy intensive operation. As a result, it is important to utilize energy efficient algorithms in achieving network wide broadcast in ad hoc networks.

There are mainly two types of approaches for energy efficient broadcast: fixed power approach and power adaptive approach. In the fixed power approach, nodes use a predetermined fixed power level for transmission. Here, the aim is to reduce power consumption by reducing the overall number of rebroadcasts during a broadcast by selecting an effective forward node set at each transmitting node [1]. In this paper the term 'rebroadcast' refers to transmission of a packet by a relay node and should not be confused with retransmission of the packet by the previous broadcasting node.

In the variable power approach, nodes use power adaptive techniques in reducing the overall power consumption in network wide broadcast. Power adaptive broadcasting and variable power broadcasting are terms used interchangeably. In the power adaptive approach, the main assumption is that the nodes can change their transmission power level dynamically. Since each node can transmit with variable power, power expenditure can be reduced by transmitting with enough power to reach only the nodes that need to be reached.

The initial proposals in power adaptive approach assume global state knowledge [2], [3]. These approaches assume that each node in the network knows the entire topology of the network in terms of connectivity between the nodes as well as the power costs of each link in the network. More recently, Cartigny et al. [4] and Chen et al. [5] proposed power adaptive broadcast algorithms that use local information. These algorithms use two hop neighborhood information where each node knows the power needed to reach its neighbors and the power needed by its neighbors to reach their neighbors. The simulation based results obtained in [5] show that the power adaptive approaches result in better energy utilization compared to fixed power approaches.

In this paper, we present a new algorithm for power adaptive broadcasting called INOP (INside-Out Power adaptive approach). INOP is designed to use two-hop neighborhood information. It utilizes a novel technique for determining the transmission power level at each node during the broadcast. Each transmitting node first sorts its neighbors based on the required power to reach them. Then, starting from the closest neighbor, the node compares the required power levels to reach the next neighbor either directly or indirectly via some other neighbor. Based on this comparison, the transmitting node decides on the transmission power level to use in its broadcast. Our simulation based comparisons with the existing state-of-the-art approaches show that our approach provides better overall performance in terms of several performance metrics. Energy efficiency indicates the average power spent in covering a node in the network. Since network wide broadcast is an unreliable operation, the coverage of all the nodes is not always guaranteed. As a result, compared to total power consumption, energy efficiency becomes a more representative metric. Additional metrics that are used in the comparisons include traffic overhead and convergence time.

The rest of the paper is organized as follows. Section II describes the energy model. Section III presents a classification of the available broadcast approaches. Section IV describes INOP, the proposed algorithm. Section V presents the simulation based evaluations and Section VI concludes the paper.

\section{ENERGy MODEL}

We consider ad hoc networks where nodes cooperate with each other to support broadcast operation. The network is modelled as a graph $G=(V, E)$ where $V$ is the set of nodes in the network and $E \subseteq V^{2}$ is the set of edges indicating the available communication links in the network. The set $E$ can be defined as follows:

$$
E=\left\{(u, v) \in V^{2} \mid P_{u v} \leq P_{\max }\right\},
$$

where $\mathrm{P}_{u v}$ is the power needed to reach node $v$ from node $u$ in a direct transmission and $\mathrm{P}_{\max }$ is the maximum power 
level of node $u$.

The channel model used follows the power law model widely used in the literature [6] :

$$
P_{\text {recv }} \propto P_{t x} / r^{n},
$$

where $\mathrm{P}_{\text {recv }}$ is the received power at a receiver, $\mathrm{P}_{t x}$ is the transmission power at the sender, $r$ is the distance between sender and receiver, and $n \geq 2$ is the power loss exponent.

Each node should be able to know the power needed to reach its neighbors. When a node transmits a packet, it records the power with which the packet is transmitted, $\mathrm{P}_{t x}$, in a field in the packet header. For the successful reception of a packet at a node, a minimum power level $\mathrm{P}_{\text {threshold }}$ is needed when the packet is received. If the power of the packet at reception is below $\mathrm{P}_{\text {threshold}}$, it is ignored. Thus, the receiving node can estimate the power needed to reach the transmitting node, based on the assumption of power symmetry in channels, as $\mathrm{P}_{\text {req }}=\left(\mathrm{P}_{\text {tx }} / \mathrm{P}_{\text {recv }}\right) * \mathrm{P}_{\text {threshold }}$.

Nodes in the network maintain neighborhood information which includes the minimum power needed to reach each of the neighbors. The total power consumption during a broadcast is given by : $P_{\text {total }}=\sum_{u \in U} \tilde{P}_{u}$, where $U$ is the set of nodes involved in broadcasts or rebroadcasts of packets and $\tilde{P}_{u}$ is the power with which node $u$ transmits the packet.

\section{ClasSIFICATION OF APPROACHES}

Fixed power broadcasting and variable power broadcasting are the two main approaches for broadcasting in ad hoc networks. In fixed power broadcasting, nodes broadcast with a predefined fixed power level which results in high power expenditure. Several algorithms have been proposed for fixed power broadcasting. These algorithms have been classified and discussed in [1]. More recently, several other methods have been introduced for fixed power broadcast [7], [8]. Since we are concentrating on the variable power approaches, we do not discuss the fixed power approaches in detail here.

Variable power broadcasting is a more recent approach for reduction in power consumption during broadcast operations in ad hoc networks. It is based on the assumption that nodes in the network can dynamically change their transmission power levels based on optimizations. There have been several methods proposed for power adaptive broadcasting in ad hoc networks. Variable power approaches may be broadly classified into global knowledge and local knowledge approaches.

\section{A. Global Knowledge Approach}

Global knowledge approach assumes that each node has global state information. In [2], the authors propose a global knowledge scheme called Broadcast Incremental Power (BIP) algorithm. The global state information at each node includes knowledge of the topology of the entire network along with the power costs of the links. In this scheme, a minimum power tree is constructed with the source node as the root of the tree. The source node includes its neighbor node which can be reached with least power into the minimum power tree. Then, each node in the tree calculates the additional power needed to reach a node not in the tree. The node that can be reached with the least additional power is then chosen to be included in the tree. This process is repeated till all the nodes are included in the tree. Finally, the source node begins the broadcast and the packets are propagated down the constructed minimum power tree. The number of rebroadcasts can be further reduced by utilizing the omnidirectional transmission of the packets. In [3], the author proves that the minimum energy broadcast tree problem in ad hoc networks is an NPComplete problem and proposes an approximation algorithm using Steiner Trees. Global knowledge methods are considered to be prohibitively expensive for practical usage as they incur a large communication overhead that is required to maintain global network knowledge in the nodes. In addition, as the network size increases, these approaches become non-scalable.

\section{B. Local Knowledge Approach}

Local knowledge approach uses local state information at each node. Each node is expected to know its neighborhood information only up to a fixed number of hops. Most of the existing approaches assume two hop neighborhood information. Each node needs to know the power needed to reach its neighbors and the power needed by its neighbors to reach their neighbors. The two well-known local knowledge based approaches include (1) a graph-based approach and (2) a centripetal approach.

\section{Graph-Based Approach}

A graph-based approach for power adaptive broadcasting in ad hoc networks is described in [4]. Here, the authors present a scheme that uses two hop neighborhood information. Each node constructs its Relative Neighborhood Graph (RNG) and attempts to choose the transmission power level so that it can reach the nodes only within its RNG. The RNG graph of a graph $G=(V, E)$ is denoted by $R N G(G)=\left(V, E_{r n g}\right)$ where $V$ is the set of nodes in the network and $E_{r n g}$ is defined as follows:

$$
\begin{gathered}
E_{r n g}=\{(u, v) \in E \mid \nexists w \in V(u, w),(w, v) \in \\
E \wedge d(u, w) \leq d(u, v) \wedge d(v, w) \leq d(u, v)\},
\end{gathered}
$$

where $d(u, v)$ is the perceived distance between nodes $u$ and $v$ in $G$. This condition is shown in Figure 1(a). In the figure, edge $(u, v)$ is not considered to be in the RNG because of node $w$. In other words, the RNG consists of all edges $(u, v) \in E$ such that there is no node $w \in V$ in the intersection of the circles centered at nodes $u$ and $v$ and radius $d(u, v)$. Each node then transmits with power enough to reach its neighbors in $\mathrm{RNG}(\mathrm{G})$.

\section{Centripetal Approach}

In centripetal (outside-in) approach, the main idea is to reduce the transmission power level by eliminating distant neighbors from the coverage set. This approach has been followed in the PABLO protocol presented in [5]. The algorithm works as follows: Consider the network where the source node $s$ has a set of neighbors $N=\{1,2, \cdots, \mathrm{k}\}$. Let $\mathrm{P}_{j}$ be the power to reach neighbor $j$ directly. Let node $k$ be the farthest neighbor of node $s, \mathrm{P}_{k}=\max \left(\mathrm{P}_{j}\right), j \in \mathrm{N}$. Node $s$ checks if node $k$ can be reached by any of $s$ 's one hop neighbors. Let node $r$ be the one hop neighbor with the least value of $\left(\mathrm{P}_{r}+\mathrm{P}_{r k}\right), r \in \mathrm{N}$. 


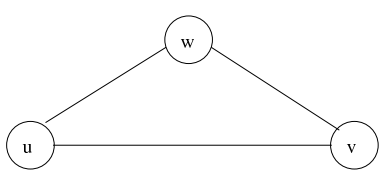

(a) Edge $(u, v)$ not included in RNG

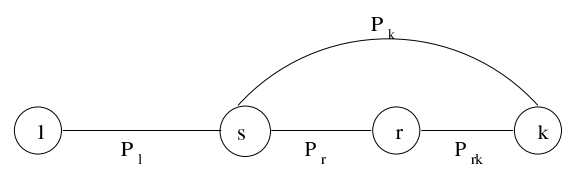

(b) Using $\mathrm{P}_{k}$ for comparison

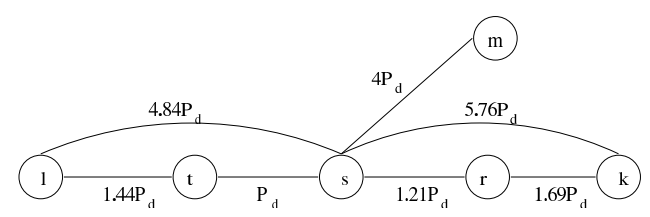

(c) Centrifugal Approach

Fig. 1. Variable Power Approaches

Find $k \in \mathrm{N}$ such that $\left(\mathrm{P}_{k}>\mathrm{P}_{i}\right) \forall i \in N-\{k\}$;

Find $r \in N-\{k\}$ such that $\left(\mathrm{P}_{r}+\mathrm{P}_{r k}\right)<\left(\mathrm{P}_{q}+\mathrm{P}_{q k}\right) \forall q \in N-\{k, r\}$ if $\left(\mathrm{P}_{r}+\mathrm{P}_{r k}\right)<\mathrm{P}_{k}$ else

Eliminate $k$ from $N ; / * k$ can be reached via $r * /$

Transmit directly to $k$;

Fig. 2. Power Adaptive Broadcasting using PABLO [5]

The scenario is as shown in Figure 1(b). In case a relay node $r$ is found, node $s$ can reduce its transmission power to $\mathrm{P}_{l}$ where node $l$ is the next farthest node after eliminating node $k$. Now, node $s$ attempts to reach node $l$ through a relay node as described earlier. This process continues till no further power reduction can be achieved. Algorithm is shown in Figure 2. There have been further improvements proposed on this approach by the authors in [9]. One improvement proposed is that the source node explicitly removes its farthest neighbor if it has a degree of two or more, after applying the above algorithm. Another improvement is that the source node removes 25 percent of its neighbors if it has a degree of four or more after applying the above algorithm. The degree of a node is the number of one hop neighbors for that node.

An observation that we make about the PABLO algorithm is that the comparison used in the algorithm (the third line in Figure 2) may not always give a good result and an alternative comparison in the form of $\left(\mathrm{P}_{l}+\mathrm{P}_{r k}\right)$ vs. $\mathrm{P}_{k}$ may result in better energy utilization especially if $\mathrm{P}_{l}>\mathrm{P}_{r k}$ and $\mathrm{P}_{k}=\mathrm{P}_{l}+\epsilon$ where $\epsilon$ is a very small value. Our simulation based preliminary evaluations show that this modification results in some small improvement in power efficiency over the original approach.

\section{INOP: A CENTRIFUGAL APPROACH}

In this section, we describe our algorithm, INOP, for variable power broadcasting. INOP uses a centrifugal (insideout) approach. In this approach, the transmitting node selects its power level by considering its neighbors starting from the closest one and moving outwards. The transmitting node computes the most probable power needed to reach the nodes either directly or using intermediate nodes. It then selects the minimum power with which to transmit so as to reduce the overall power consumption in covering all one-hop neighbors. The detailed algorithm is presented below.

In INOP, a transmitting node $s$ computes the most probable power to reach each neighbor node $p, \mathrm{P}_{p}^{p r o b}$. The transmitting node $s$ starts from $n$, the nearest node. In order to reach $n, s$ has to transmit directly. So, the most probable power to reach node

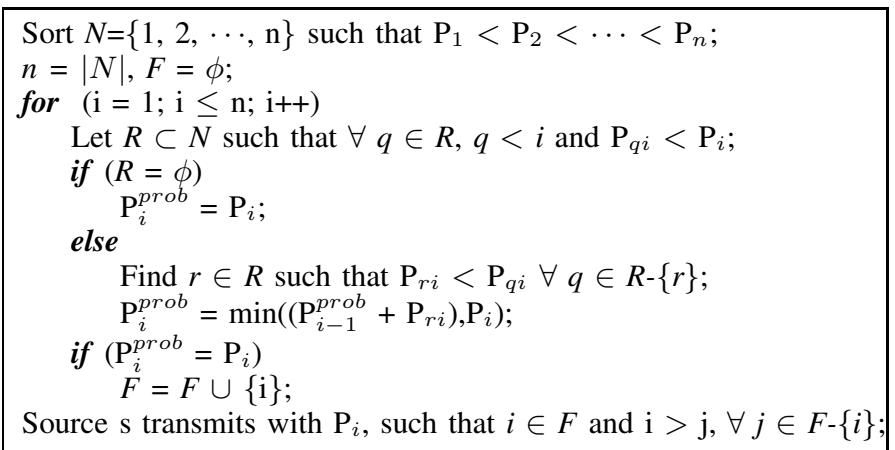

Fig. 3. INOP Approach

$n$ is $\mathrm{P}_{n}^{p r o b}=\mathrm{P}_{n}$. When $s$ transmits with a minimum power of $\mathrm{P}_{n}$, the neighbor $n$ is said to be covered by that transmission. Next, the most probable power to reach the second nearest node $m$ is calculated as the minimum of (1) the power to reach $m$ directly from node $s$ and (2) the sum of the most probable power to reach node $n, \mathrm{P}_{n}^{p r o b}$, and the power to reach $m$ from the only intermediate node $n$. In general, the most probable power to reach any node $i$ is computed as follows. Let the most probable power to reach the previous nearest node $h$ be $\mathrm{P}_{h}^{p r o b}$. Let $r$ be a covered node that has the least $\mathrm{P}_{r i}$ value. The minimum of $\mathrm{P}_{h}^{p r o b}+\mathrm{P}_{r i}$ and $\mathrm{P}_{i}$ is assigned to $\mathrm{P}_{i}^{p r o b}$. The value of $\mathrm{P}_{i}^{p r o b}$ is calculated for all $i \in N$. Finally, the transmitting node $s$ transmits with enough power to reach node $p$, where $p$ is the farthest node with $\mathrm{P}_{p}^{p r o b}=\mathrm{P}_{p}$. The algorithm is shown in Figure 3.

We expect INOP to perform better than the earlier approaches as it considers the most probable power to reach a node during broadcast. For example, consider the scenario shown in Figure 1(c). PABLO described above will not ensure reduced power consumption. According to PABLO, in this particular example case, based on the comparison $\left(\left(\mathrm{P}_{r}+\mathrm{P}_{r k}\right)=2.9 \mathrm{P}_{d}\right)<\left(\mathrm{P}_{k}=5.76 \mathrm{P}_{d}\right)$, node $k$ will be eliminated. Then, since $\left(\left(\mathrm{P}_{t}+\mathrm{P}_{t l}\right)=2.44 \mathrm{P}_{d}\right)<\left(\mathrm{P}_{l}=4.84 \mathrm{P}_{d}\right)$, node $l$ will also be eliminated. But node $m$ cannot be eliminated as there is no intermediary node to reach it. So, the total power consumption for the entire broadcast will be $\left(\mathrm{P}_{m}+\mathrm{P}_{r k}+\mathrm{P}_{t l}\right)=7.13 \mathrm{P}_{d}$, which is much higher than the power to reach node $k$ directly.

In the case of INOP, the algorithm starts from the closest node $t$ and moves outward. Based on the algorithm presented in Figure 3, the source node $s$ chooses to transmit with a power level of $5.76 \mathrm{P}_{d}$ to reach $k$ directly. Thus, INOP consumes the optimal power in this situation. This is because in INOP, the 
transmitting node $s$ considers each neighbor by moving from the nearest node to the farthest node and calculates the most probable power level to cover each of them.

All of the broadcast approaches presented so far aim at optimizing power expenditure in covering the nodes that are in the transmission range of the broadcast originating node $s$, i.e., first hop neighbors of $s$. Let $C$ be the set of nodes covered by the broadcast originating node directly. Let $\bar{C}=N-C$. The overall performance of a broadcast approach partly depends on how to choose a set of rebroadcast nodes to cover the remaining nodes (including the uncovered first hop neighbors of $s$ and others) in the network. This problem is known as neighbor selection problem and is known to be an NPcomplete problem [10]. In the current version of our work, we use a heuristic that is used in PABLO for neighbor selection. As part of our future studies, we will work on improving the performance of our algorithm by developing more efficient neighbor selection heuristics.

The neighbor selection approach that we use in our algorithm works as follows. Consider a node $n$ that is covered by the broadcast of some node $s$. Let $\operatorname{NSet}(n)$ represent the set of neighbors for node $n$. When $n$ receives a broadcast packet from $s$, it calculates a Rebroadcast Backoff Timer (RBT) and delays its rebroadcast for RBT seconds. RBT is randomly selected from an interval that is inversely proportional to the number of uncovered neighbors of $n$. The main goal of this delay interval is to reduce collisions and avoid redundant rebroadcasts by allowing nodes that have a larger set of uncovered neighbors to rebroadcast first. During RBT seconds, $n$ listens to the channel for rebroadcasts coming from other covered neighbors of $s$. At the end of RBT seconds, $n$ identifies set of its neighbors, $\operatorname{CNSet}(n)$, that must have been covered by the broadcast of $s$ or by the rebroadcasts of some of the neighbors of $s$. If the set $\operatorname{NSet}(n)-\operatorname{CNSet}(n)$ is empty, $n$ cancels its rebroadcast. If not, it uses INOP approach to determine its transmission power level and rebroadcasts the packet.

\section{Performance Evaluations}

In this section, we present our simulation based comparisons between INOP and several other broadcast algorithms. We first describe our simulation environment and the comparison metrics used in the evaluations. The simulation parameters used in our evaluations are described in Table I. In each simulation, one of the nodes is randomly selected to be the broadcast originator node. This node generates periodic broadcast packets every second until the end of simulation time. Each broadcast packet is marked by a sequence number that helps us distinguish different broadcast packets from each other. In the current version of the evaluations, we do not consider unicast-based cross traffic in the network. Having such cross traffic will likely affect the coverage characteristic of alternative approaches. In our future work, we will consider scenarios where we have unicast based cross traffic in the network and study the performance of alternative broadcast approaches in that context.

Parameters considered for evaluation of the algorithms are:
TABLE I

Simulation Parameters

\begin{tabular}{|c|c|}
\hline Parameter & Value \\
\hline \hline Simulator & NS-2 \\
\hline Simulation Area & $500 \times 500$ meter \\
\hline Node Transmission Range & 100 meter \\
\hline Simulation Time & 10 seconds \\
\hline
\end{tabular}

- Energy Efficiency: Energy efficiency is defined as energy spent per covered node and is calculated as the ratio of the total power consumption to the number of covered nodes. Energy efficiency indicates the average power spent in reaching any covered node in the network. Broadcast in ad hoc networks is an unreliable operation and the coverage of all the nodes is not guaranteed. As a result, compared to total power consumption, energy efficiency is a more representative metric to compare the performance of different broadcast approaches.

- Coverage: This metric indicates the degree of coverage provided by different protocols.

- Num. of Rebroadcasts: The number of rebroadcasts indicates the traffic generated in the network. With each transmission, there is a possibility for collision which may affect the network capacity. Therefore, number of rebroadcasts is a useful metric for comparison.

- Convergence Time: Convergence time is the time period from the start of the broadcast operation at the source node up to the time when the last node receives the broadcast packet.

\section{A. Comparisons of Variable Power Broadcast Approaches}

In this subsection, we present our simulation based comparison results on the performance of the variable power broadcast algorithms including BIP, RNG, PABLO, and INOP. BIP uses global knowledge of the network topology and achieves an optimal performance in terms of energy efficiency. However, due to the difficulties of maintaining topology knowledge, it is considered to be impractical. In our evaluations, we use BIP as a yardstick to compare the other three approaches, especially in terms of their energy efficiency. We use 20, 40, 60,80 , and 100 node topologies for our simulations. For each topology size, we ran simulations on 20 different topologies and present the average values of the results.

The energy efficiency of the approaches is compared in Figure 4(a) where we present the relative error of approximation of INOP, PABLO, and RNG approaches with respect to the optimal approach BIP. For each data point, we compute the relative error as relative error $=(x-y) / y$, where $x$ is the energy efficiency of an approach (INOP, PABLO, or RNG) and $y$ is the energy efficiency of BIP. According to the figure, in the case of 20 node networks, INOP gives its best performance. It performs within $16 \%$ of the optimal whereas PABLO performs within $37 \%$ of the optimal. On the other hand, the worst performance of INOP and PABLO approaches are seen at 60 node network case where INOP performs within $33 \%$ of the optimal and PABLO performs $42 \%$ of the optimal. The results 


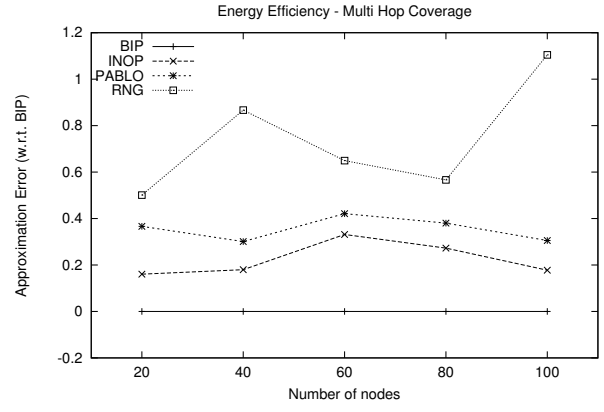

(a) Energy Efficiency (w.r.t. BIP) vs. Network Size

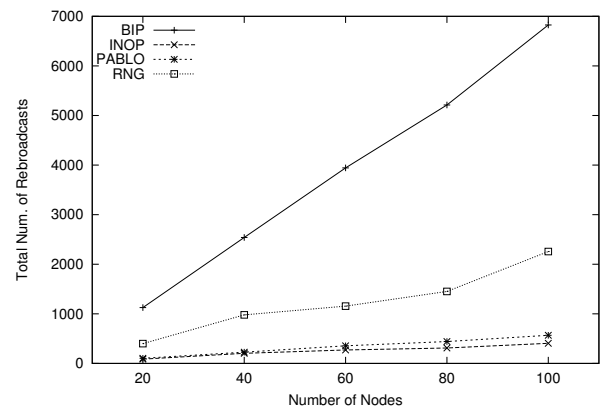

(c) No. of Rebroadcasts vs. Network Size

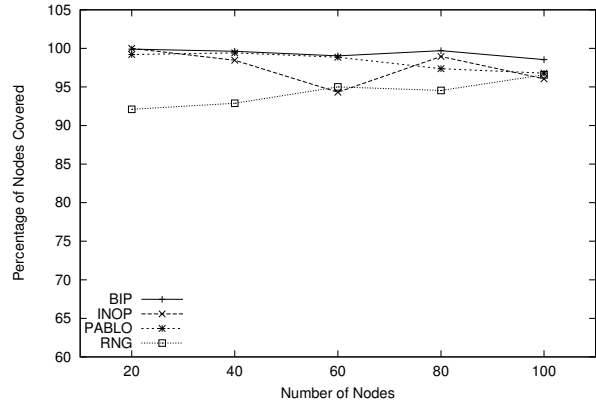

(b) Percentage of Nodes Covered vs. Network Size

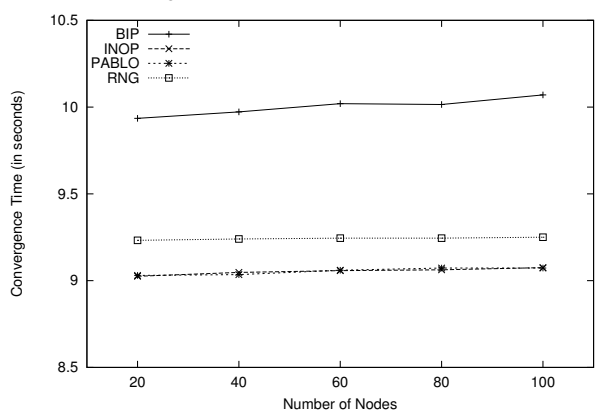

(d) Convergence Time vs. Network Size

Fig. 4. Comparison of variable power approaches

presented in this figure show that the relative performance of INOP is consistently better than the other approaches.

Figure 4(b) shows that the approaches have similar coverage with BIP providing the best coverage, followed by PABLO and INOP. The coverage of RNG seems to be the worst among the alternative approaches but it still remains above $90 \%$. Number of rebroadcasts is an indication of the traffic overhead introduced into the network during a network wide broadcast. Considering the overall network throughput, the lesser the number of rebroadcasts, the better the overall network throughput. Figure 4(c) compares the approaches based on the number of rebroadcasts. According to the figure, INOP uses the least number of rebroadcasts and is closely followed by PABLO. In addition, the figure shows a trend that as the network gets denser the relative performance of INOP gets better compared to other approaches. This is desirable as it also reduces contention for medium access and collisions of transmitted packets. The comparison of the convergence times of the different algorithms are presented in Figure 4(d). As expected, BIP has the highest convergence time, while INOP and PABLO perform better than the other approaches.

In summary, INOP has important advantages in terms of energy efficiency and performs as good as or better than the others in terms of its coverage, traffic overhead (i.e., number of rebroadcasts), and convergence time. Considering the energy efficiency as the primary motivation in variable power broadcast approaches, INOP clearly improves on the existing approaches without sacrificing on the other metrics commonly used to evaluate network wide broadcast algorithms.

\section{B. Comparisons to Fixed Power Broadcast Approaches}

In this subsection, we compare INOP with one of the best fixed power broadcast approaches, namely, the Ad Hoc Broadcast Protocol (AHBP) [11]. Figure 5 presents the results for energy efficiency and number of rebroadcast. Both approaches have almost the same coverage and convergence time (figures not shown). According to figures, on the average, AHBP requires about 2.5 times more energy as compared to INOP. Also, even though INOP requires significantly larger number of rebroadcasts, this does not necessarily indicate a significant disadvantage compared to AHBP. This behavior can be attributed to the very nature of variable power broadcast approaches where each node dynamically adjusts its power range so as to improve power savings. As seen earlier, INOP has the least number of rebroadcasts among the variable power approaches and a significant gain in energy efficiency with only local knowledge. Compared to AHBP, it requires a larger number of rebroadcasts but each rebroadcast uses much less energy and therefore covers much less area. This helps reduce the possibility of collisions with competing traffic. In addition, both INOP and AHBP have similar behavior in terms of coverage and convergence time indicating that the difference in the number of rebroadcasts does not necessarily indicate a disadvantage for the approach. As a result, we can conclude that our approach takes the advantage of power adaptive techniques in order to improve the energy efficiency and at the same time reduces the traffic overhead in the network as in the case of fixed power approaches. 


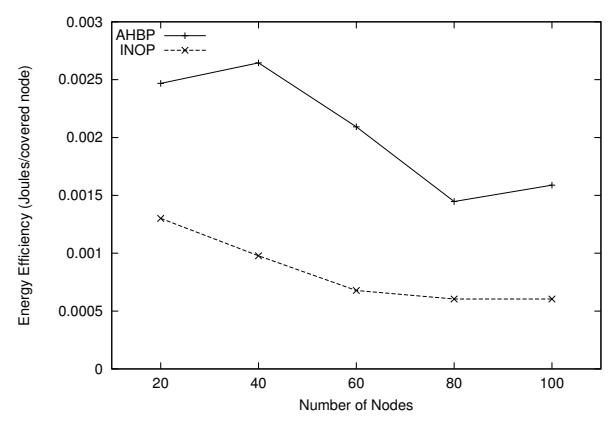

(a) Energy Efficiency vs. Network Size

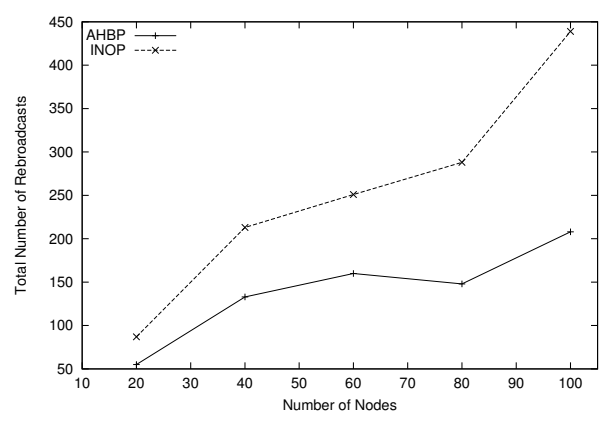

(b) Number of Rebroadcasts vs. Network Size

Fig. 5. Comparison of INOP and AHBP

TABLE II

COMPARISON OF THE PROPERTIES OF THE TREES

\begin{tabular}{|c|c|c|c|}
\hline Algr. & $\begin{array}{c}\text { Avg. } \\
\text { outdegree }\end{array}$ & $\begin{array}{c}\text { Avg. trans. } \\
\text { range }\end{array}$ & Depth \\
\hline \hline INOP & 2.1 & $36.7 \mathrm{~m}$ & 14 \\
\hline PABLO & 1.6 & $34.9 \mathrm{~m}$ & 18 \\
\hline RNG & 1.6 & $33.4 \mathrm{~m}$ & 20 \\
\hline BIP & 1.6 & $31.8 \mathrm{~m}$ & 24 \\
\hline
\end{tabular}

\section{Comparison of Broadcast Trees}

In this section, we use a sample simulation case to examine the characteristics of broadcast trees built by alternative approaches. This study helps us to understand the characteristics of different algorithms considered in this paper in a better way. We use the results of a simulation case where an 80 node network topology is used to generate broadcast trees formed by different algorithms.

Average outdegree, average transmission range, and depth of the tree are interesting aspects that can be compared among the trees. Outdegree of a node refers to the number of children that the node has on the tree. Average outdegree gives an indication of the number of rebroadcasts used in the broadcast operation. If average outdegree is high, it indicates that a large number of neighbors are covered with each rebroadcast. Hence, the number of rebroadcasts will be lesser. The average transmission range provides information about the strength of broadcast at each node. If this value is high, then the nodes broadcast with a higher power, covering a higher number of nodes in a single broadcast. Depth of the tree is the number of hops from the source node to the farthest node in the tree. The higher the depth of the tree is, the higher is the number of rebroadcasts and the higher is the convergence time. Table II provides a comparison of these parameters for the four trees shown. Our overall observation from these results is that, compared to other approaches, in INOP, nodes use larger transmission ranges to cover more number of nodes that results in broadcast trees with smaller depths.

\section{CONCLUSION}

In this paper, we have presented a new algorithm for variable power broadcast in ad hoc networks. Initially, we have pre- sented a classification of existing variable power broadcast algorithms based on their main approaches as global knowledgebased, graph-based, and centripetal approaches. We have then introduced centrifugal approach as a more promising approach and developed our algorithm, INOP, using this approach. We have presented simulation based comparisons of INOP with the existing set of variable power approaches (BIP, PABLO, and RNG) and a representative fixed power broadcast approach (AHBP). Our evaluations have shown that compared to other variable power approaches, INOP achieves better results in terms of energy efficiency, and competes and exceeds other approaches in terms of a number of other performance metrics including traffic overhead, coverage, and convergence time.

\section{REFERENCES}

[1] B. Williams and T. Camp, "Comparison of broadcasting techniques for mobile ad hoc networks," in Proceedins of MOBIHOC, Lausanne, Switzerland, June 2002.

[2] J. Wieselthier, G. Nguyen, and A. Ephrimides, "On construction of energy-efficient broadcast and multicast trees in wireless networks," in Proceedings of IEEE INFOCOM, Tel Aviv, Israel, March 2000.

[3] W. Liang, "Constructing minimum-energy broadcast trees in wireless ad hoc networks," in ACM International Symposium on Mobile Ad Hoc Networking and Computing, Lausanne, Switzerland, June 2002.

[4] J. Cartigny, D. Simplot, and I. Stojmenovic, "Localized minimum-energy broadcasting in ad-hoc networks," in Proceedings of IEEE INFOCOM, San Francisco, CA, USA, March/April 2003.

[5] X. Chen, M. Faloutsos, and S. Krishnamurthy, "Power adaptive broadcasting with local information in ad hoc networks," in Proceedings of IEEE ICNP, Atlanta, GA, USA, November 2003.

[6] T. Rappaport, Wireless Communications, Principles and Practices. Prentice Hill, 1996.

[7] M. Agarwal, J. H. Cho, L. Gao, and J. Wu, "Energy efficient broadcast in wireless ad hoc networks with hitch-hiking," in Proceedings of IEEE INFOCOM, Hong Kong, March 2004.

[8] W. Lou and J. Wu, "Double-covered broadcast (dcb): A simple reliable broadcast algorithm in manets," in Proceedings of IEEE INFOCOM, Hong Kong, March 2004.

[9] K. Karenos, A. Khan, X. Chen, M. Faloutsos, and S. Krishnamurthy, "Local versus global power adaptive broadcasting in ad hoc networks," in Proceedings of IEEE WCNC, New Orleans, MI, USA, March 2005.

[10] A. Qayyum, L. Viennot, and A. Laouiti, "Multipoint relaying for flooding broadcast messages in mobile wireless networks," in Proceedings of 35th Havaii International Conference on System Sciences, Big Island, HI, USA, January 2002.

[11] W. Peng and X. Lu, "Ahbp : An efficient broadcast protocol for mobile ad hoc networks," in Journal of Science and Technology, Beijing, China, 2002. 